\title{
GLAD!
}

Revue sur le langage, le genre, les sexualités

$07 \mid 2019$

Varia

\section{Guillaume Marche. 2017. La militance LGBT aux États- Unis : sexualité et subjectivité}

Juliette Melia

\section{OpenEdition}

1 Journals

Édition électronique

URL : http://journals.openedition.org/glad/1725

DOI : $10.4000 /$ glad. 1725

ISSN : 2551-0819

Éditeur

Association GSL

Référence électronique

Juliette Melia , "Guillaume Marche. 2017. La militance LGBT aux États-Unis : sexualité et subjectivité », GLAD! [En ligne], 07 | 2019, mis en ligne le 05 décembre 2019, consulté le 17 décembre 2020. URL : http://journals.openedition.org/glad/1725; DOI : https://doi.org/10.4000/glad.1725

Ce document a été généré automatiquement le 17 décembre 2020.

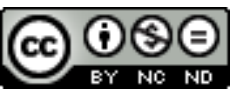

La revue GLAD! est mise à disposition selon les termes de la Licence Creative Commons Attribution Pas d'Utilisation Commerciale - Pas de Modification 4.0 International. 


\title{
Guillaume Marche. 2017. La militance LGBT aux États-Unis : sexualité et subjectivité
}

\author{
Juliette Melia
}

\section{RÉFÉRENCE}

Guillaume Marche. 2017. La militance LGBT aux États-Unis : sexualité et subjectivité, Presses Universitaires de Lyon, collection SXS Sexualités.

\section{Introduction}

1 Le combat pour les droits des homosexuelle·s américain'e-s a-t-il été gagné en 2015, quand iels ont gagné, de haute lutte, la légalisation du mariage entre personnes du même sexe? N'est-il pas paradoxal que les gays aient dû minimiser leur différence sexuelle pour obtenir des droits pour leur communauté ? Peut-il y avoir des avantages politiques à la terrible épidémie du sida? Pourquoi les militantees se sont-iels détournée's des organisations LGBTQ ? L'infrapolitique et la contre-culture pourrontelles réenchanter la militance LGBT ? Comment s'articule la dialectique entre discours militants et discours institutionnels? Voici quelques-unes des questions que pose le livre de Guillaume Marche, La Militance LGBT aux États-Unis: Sexualité et Subjectivité, publié en 2017 aux Presses Universitaires de Lyon dans la collection SXS Sexualités. L'ouvrage est composé de deux parties. La première (les deux premiers chapitres) reprend l'histoire de la militance LGBT, de l'après Seconde Guerre mondiale à la fin des années 1990. La deuxième (les trois suivants) revient sur ces éléments historiques par le biais des entretiens sociologiques. Ainsi, les discours historiques et politiques s'allient aux discours personnels sur la sexualité pour explorer la subjectivité du vécu homosexuel annoncée dans le titre. 
2 Guillaume Marche s'appuie sur une double expertise : d'une part, une connaissance du terrain acquise à partir des années 1990, d'autre part, un travail théorique de sociologue et d'historien. C'est ce double éclairage qui fait l'intérêt de ce livre. En effet, il pose des questions, dont certaines peuvent sembler inattendues, voire mineures, à qui n'est pas confronté aux discriminations que ces questions révèlent. Il s'attache ensuite à montrer leur force politique, en y apportant des éléments de réponses qui s'appuient sur l'histoire, la théorie sociologique, et des entretiens avec des membres d'associations politiques, militantes ou socioculturelles LGBT. En effet, Guillaume Marche souligne dès son introduction que son étude porte sur la tendance du mouvement qui voit dans les luttes pour les droits des homosexuel.le.s une possibilité de transformer radicalement la société américaine, ce qui explique son choix d'interlocuteurs.

3 L'auteur s'appuie également sur différents matériaux langagiers (entretiens, mais aussi autocollants, graffiti, documents d'archives) qui éclairent à quel point l'histoire se fait à partir d'un entremêlement discursif. Il en résulte un ouvrage qui ne traite pas à proprement parler des problématiques du langage, mais qui montre comment différents discours s'organisent pour donner du sens à des situations sociales concrètes.

\section{Grands mouvements de la militance gaie}

4 La Militance LGBT s'ouvre sur un rappel des principaux discours historiques concernant les mouvements pour les droits des homosexuels.

5 Au sortir de la Seconde Guerre mondiale et de ses bouleversements sociaux, en 1950 à Los Angeles, Harry Hay crée la Mattachine Society, «première organisation politique fondée sur l'identification commune d'homosexuels $»^{1}$. Puis, en 1955, Del Martin et Phyllis Lyon fondent l'équivalent lesbien, les San Francisco Daughters of Bilitis. Dans le contexte du maccarthysme, les homosexuel.le.s doivent en effet s'organiser contre la chasse aux sorcières dont iels sont victimes. Très vite, dès 1953, Mattachine évolue vers l'assimilationnisme, ses nouveaux membres souhaitant minimiser l'importance de leur homosexualité dans leur construction identitaire. Mais les autres activités de l'association, par exemple de publication et d'aide psychologique et juridique aux homosexuel.le.s, font évoluer les mœurs en profondeur malgré l'assimilationnisme de surface qui désamorce le potentiel militant et radical de l'homosexualité.

6 À partir de la seconde moitié des années 1960, la culture gaie est associée à la culture des bars et se sexualise de nouveau. On assiste par exemple à la naissance des mouvements camp et butch-femme, deux façons de se réapproprier l'insulte avec fierté, voire excès, dans un esprit d'antiparastase (Erving Goffman parle de « retournement du stigmate »). C'est dans cette époque de culture des bars qu'éclatent les émeutes du Stonewall Inn le 27 juin 1969. La même année et à New York aussi, le GLF, Gay Liberation Front, est créé et organise des événements qui théâtralisent la sexualité afin de s'affranchir de l'oppression de la norme.

7 Puis, dans les années 1970, malgré quelques efforts vers le libérationnisme (transformation des normes sexuelles pour l'ensemble de la société) et l'égalitarisme (reconnaissance des droits des homosexuel.le.s), la tendance qui s'affirme avec le plus de force est celle du communautarisme, qui favorise le développement d'enclaves gaies dans les grandes villes, avec associations, mais aussi commerces et logements dédiés. Le 
résultat est la désexualisation, et par conséquent la dépolitisation, des identités homosexuelles. Le but recherché en se regroupant ainsi est finalement de vivre « comme tout le monde » et non plus de réinventer une société subversive.

8 L'épidémie du sida, au début des années 1980, marque une évolution dans les discours à propos de l'homosexualité. Les débats qui l'accompagnent sont empreints de paradoxes : ils renforcent l'idée reçue que l'homosexualité est une maladie, tout en consolidant la cohésion du groupe. D'une part l'épidémie débouche sur un aggravement de la stigmatisation de l'homosexualité, et d'autre part, si les hommes gais étaient les plus touchés, les lesbiennes prennent largement en charge le soutien aux malades. À nouveau, la dynamique est celle de l'assimilationnisme et de la désexualisation, perçus alors comme le meilleur moyen de ne pas s'aliéner le moindre financement pour la recherche et la prévention. C'est en réaction à cette institutionnalisation désexualisante qu'est créé Act Up en 1987 à New York. Nous sommes dans un contexte d'influence de la droite chrétienne, et c'est aussi contre les discours moralisateurs des Républicains Ronald Reagan et Jesse Helms que se déploient les actions contreculturelles et théâtrales d'Act Up (perturbation de la Bourse à Wall Street le 24 mars 1987 afin de protester contre les profits générés par les traitements antisida, die-in, kissin, et même tit-in...) qui rendent publiques et par conséquent politiques des sexualités que les pouvoirs conservateurs voudraient rendre taboues à défaut de pouvoir les éradiquer.

9 L'élection de Bill Clinton à la Maison Blanche en 1993 se fait avec le soutien des homosexuel.le.s, à qui il promet durant sa campagne électorale l'accès aux carrières militaires. En 1994, un accord est conclu, c'est la doctrine du « Don't Ask, Don't Tell » : les homosexuel.le.s ont le droit de servir dans l'armée, les recruteurs n'ont pas le droit de leur poser des questions sur leur orientation sexuelle, mais eux-mêmes n'ont pas le droit d'en parler. Mais ce système peut s'avérer délétère pour les militaires gai.e.s ainsi condamné.e.s à un éternel "placard", car il définit l'homosexualité comme une conduite (comportement choisi) alors que le consensus des militant.e.s pour les droits LGBT est de la considérer comme un statut (condition involontaire et innée).

10 La dernière partie du premier chapitre rappelle les étapes de la reconnaissance du mariage homosexuel, sans occulter le paradoxe qui consiste à faire de cette victoire, en 2015, une défaite pour les homosexuel.le.s qui ne souhaitent pas se reconnaitre dans cette institution patriarcale, monogame et hétéronormée. Le deuxième chapitre, intitulé « Entre éclatement et coalescence », reprend plus en détail la période allant des années 1980 aux années 1990 pour expliquer comment la désexualisation de l'homosexualité, qui a fini par caractériser la fin des années 1990, est la cause profonde de la désaffection militante observée de nos jours sur le terrain.

\section{Micro-politique gaie}

11 Le troisième chapitre, " Épanouissement et déception ", montre comment les militants revendiquent dans leurs prises de paroles la dimension politique de leur action, et inclut également des remarques de terrain. En effet, la montée du mercantilisme arcen-ciel des quartiers homosexuels autrefois « underground » n'est perceptible que pour qui prend le pouls de ces quartiers depuis plusieurs décennies et comprend leur mutation. Un des sous-groupes les plus touchés par ces évolutions est la communauté sadomasochiste et cuir initialement regroupée dans le quartier South of Market de San 
Francisco. On la soupçonne d'accélérer la propagation du virus du sida, et la municipalité soutient donc une mutation du quartier en favorisant l'implantation de commerces tournés vers des populations jeunes, riches et hétérosexuelles. Le Folsom Street Fair perdure, ce festival de rue qui permet la visibilité et la sociabilité des pratiquants SM, mais il devient de plus en plus folklorique, avec des spectateurs extérieurs à la communauté et donc potentiellement voyeurs.

Dans ce contexte, Guillaume Marche revient sur les polémiques qui troublent les Marches des Fiertés depuis le milieu des années 1990. En 1997, la soirée qui suit la Marche est payante et très chère, excluant de fait les populations aux revenus modestes. En 1998, les organisateurs installent des barrières le long du parcours, créant une séparation symbolique entre participant·e's et spectateur.trices. Tous les sponsors n'ont pas des attitudes irréprochables en matière de lutte contre les discriminations. Chelsea Manning, femme transsexuelle, associée au site WikiLeaks, n'est pas autorisée à être invitée d'honneur de la Marche de 2013 pour des raisons politiques. L'image qui se dessine de cette succession de malaises est que la commémoration des émeutes de Stonewall et de ce qu'elles ont représenté pour les droits des homosexuel-le·s s'efface devant la force de la société de consommation.

La désexualisation progressive des Marches des Fiertés se retrouve sur le plan personnel dans la vie des homosexuellle·s, à qui il est demandé d'occulter tout sentiment négatif (honte, culpabilité vis-à-vis de l'épidémie du sida, dégout de sa sexualité...). Le modèle, en quelque sorte, est la comédienne Ellen DeGeneres, qui fait son coming-out en même temps que son personnage de sitcom, repoussant il est vrai une limite, mais sans image choquante pour un public qu'elle aurait pu ainsi s'aliéner: aucune image d'intimité physique entre femmes n'est montrée à l'écran. Les homosexuel-le·s sentent qu'ils doivent se conformer à des attentes hétéronormées qui ne leur correspondent pas. Se conformer à une norme équivaut à renoncer à ce que Guillaume Marche appelle l'authenticité, qui permet d'atteindre une véritable réalisation de soi par le mécanisme suivant : «l'authenticité peut donc nécessiter de la part de l'individu la prise d'un risque : celui de se dévaloriser, en adoptant par exemple une identité disqualifiée afin d'exposer un rapport de pouvoir et de la contester. ${ }^{2}$ Sans authenticité, pas de politique possible, et donc pas d'égalité non plus.

\section{Réenchanter le politique}

Les deux derniers chapitres s'attachent à trouver des modes de militance qui remettent au premier plan les subjectivités et les sexualités des homosexuel-le·s déçu·e's par la victoire à la Pyrrhus que représente l'accès au mariage, puisqu'il semble sacrifier le potentiel radical des relations sexuelles gaies, par la perte de sens des Marches des Fiertés, par l'aseptisation de l'image des homosexuel.le.s dans les médias, et enfin par l'institutionnalisation désexualisante des mouvements militants gais. Les trois modes d'action principaux sont la mise en place de groupes de rencontres entre homosexuel.e.s (par exemple entre jeunes, que les risques d'accusation de pédophilie excluent des discours officiels), la subversion des manifestations officielles (scandale des Lesbian Avengers, qui en 1996 à Boston fait défiler un char équipé d'un lit où des lesbiennes simulent des actes sexuels), ou les pratiques contre-culturelles (association LaGAI qui propose des actions participatives et théâtralisées sur un mode humoristique mais n'excluant pas le politique). Le principe de plaisir est une fin en soi pour ces 
actions dont les participante-s peuvent se rencontrer et s'amuser afin de construire leur propre authenticité.

Le dernier chapitre décrit et analyse des actions infrapolitiques, « en deçà de ce qui est normalement considéré comme légitimement politique $»^{3}$. Le théâtre est la première forme d'action abordée, qui permet par exemple de transmettre des messages aux spectateurstrices sur les pratiques sexuelles à moindre risque, sans stigmatisation puisque les comédien'ne's, eux-mêmes homosexuel·le's, sont impliquée's de fait dans le propos.

Guillaume Marche analyse ensuite des modes d'action qui mobilisent des médias mineurs, mais dont l'auteur nous fait envisager la valeur radicale: la vignette photocopiée, l'autocollant, l'affichette ou le pochoir, qui politisent les murs des villes. En effet, un collectif informel crée, avec très peu de moyens, des campagnes contre l'oppression, la marchandisation des identités gaies, les violences policières et/ou l'intersectionnalité. Un mode d'action à ce point subalterne est peut-être voué à l'échec politique : ce n'est pas par l'intermédiaire du graffiti que les lois sont modifiées. De plus, les revendications inscrites sur les murs sont parfois confuses ou contradictoires, et de source indéterminée. Il s'agit donc d'une action infrapolitique, source probable d'empowerment dont le potentiel politique reste à déterminer précisément.

17 Les Sœurs de la Perpétuelle Indulgence ont, elles aussi, des actions militantes infrapolitiques où l'humour et le camp, loin de disqualifier leur action, est leur mode opératoire et fait leur force. Une dynamique similaire se retrouve chez les Church Ladies for Choice, groupe qui se rassemble devant les cliniques où sont pratiquées des IVG pour s'opposer, par l'humour, le travestissement, et les hymnes religieux aux paroles réécrites comme des chansons paillardes, aux manifestations contre le droit à l'avortement.

Guillaume Marche présente donc la contre-culture et l'infrapolitique comme des façons de réinventer une action politique subjective et créative, propre à redonner envie de militer à quiconque se sent concernéee par les droits des homosexuelle·s.

\section{Conclusion et synthèse}

Guillaume Marche revient en conclusion sur les reculs des droits des gays qui ont défrayé la chronique dans les premières décennies du XXIème siècle: controverse de l'accès aux toilettes publiques pour les personnes transgenres en 2016, renforcement en 2017 de la loi de liberté religieuse qui permet aux individus ou aux entreprises de refuser leur services à des mariages homosexuels, discriminations dans de nombreux domaines tels que l'emploi, le logement, ou la santé, qui exposent particulièrement les jeunes à l'errance, à la violence et au harcèlement. Ces reculs rendent compte de l'urgence, malgré l'impression de victoire qu'a donné le droit au mariage, à inventer des formes de militance dans lesquelles se reconnaitront les générations futures.

L'ouvrage de Guillaume Marche fait s'entrecroiser les discours historiques et militants pour montrer que les luttes pour les droits des homosexuelles ne se limitent pas à des victoires symboliques comme l'accès au mariage ou aux carrières militaires, mais qu'un accès à des droits réellement et profondément égaux aurait pu réinventer une société foncièrement plus égalitaire, plus progressiste, et moins consumériste. Sa lecture 
permettra de comprendre le passé des luttes afin de s'en inspirer et de se galvaniser pour les luttes à venir.

\section{NOTES}

1. Marche, p. 31.

2. Marche, p. 141.

3. Marche, p. 215.

INDEX

Thèmes : Actualités

Keywords : Keywords : LGBT, activism, infrapolitical, desexualization, marriage

Mots-clés : LGBT, militance, infrapolitique, désexualisation, mariage

\section{AUTEURS}

\section{JULIETTE MELIA}

Juliette Melia a rédigé une thèse (2017) sur la photographie contemporaine. Sa recherche porte principalement sur les politiques de représentation du corps et de la différence dans l'art. Elle enseigne l'anglais et la littérature anglaise en lycée, et la représentation du corps dans l'art et l'écriture créative à l'université. 